\title{
User Centered Design to incorporate predictive models for Type 2 Diabetes screening and management into professional decision support tools: preliminary results.
}

\author{
G. Fico ${ }^{1}$, IEEE and IFMBE member, L. Hernandez ${ }^{1}$, J. Cancela ${ }^{1}$ and M.T. Arredondo ${ }^{1}$ \\ ${ }^{1}$ Universidad Politécnica de Madrid/Life Supporting Technologies, Madrid, Spain
}

\begin{abstract}
Type 2 Diabetes screening and risk stratification tools could benefit from the incorporation of predictive systems based on computer modelling. The adoption of User Centered Design techniques is fundamental in order to integrate these systems in an effective and successful way. The work presented in this paper describe the methodologies used in the context of a multidisciplinary research project and provides an overview of the preliminary results.
\end{abstract}

Keywords - User Centered Design, Predictive Modelling and Data Mining, Diabetes Screening and Risk Stratification, Decision Support Tools.

\section{INTRODUCTION}

Type 2 Diabetes Mellitus (T2DM) is the most common form of diabetes. The World Health Organization estimates that by 2030 there will be about 550 million people suffering this disease [1.]. It is characterised by insulin resistance and relative insulin deficiency, either of which may be present at the time that diabetes becomes clinically manifest. The diagnosis of type 2 diabetes usually occurs after the age of 40 or even earlier, especially in populations with high prevalence of this disease. It is often associated with obesity, which itself can cause insulin resistance and lead to elevated blood glucose levels [1.][2.]. This disease can remain undetected for many years because hyperglycemia develops gradually and at earlier stages is not severe enough for the patient to notice any of the classic symptoms of diabetes. Nevertheless, such patients are at increased risk of developing macrovascular (mainly stroke and acute coronary syndromes) and microvascular (mainly retinopathy, neuropathy, nephropathy, and limb ischemia) complications and diagnosis is often made from associated complications or incidentally through an abnormal blood or urine glucose test. Risk prediction models (for both onset and evolution of the disease) have the potential to contribute to the healthcare interventions and decision-making processes, as recognized by the American Diabetes Association Consensus Panel on September 2004 [3.]. However, despite the large number of models being developed and the increased interest and acknowledgement in the clinical field, only a very small minority ends-up being used in clinical practice.

The main objective of the MOSAIC project is to develop a set of tools that will improve diagnosis, assessment and management of diabetes. These new tools are of technological nature implementing a series of models and algorithms based on medical and scientific knowledge. One of the most important challenges of the project is to combine the research activities related to the discovery of new risk factors, methods and models for diabetes onset, progression and evolution, with the integration of such risk factors, methods and models into software tools, components and modules that would incorporate these innovations and make them usable by different end-users in different settings and for different purposes. In other words, the challenge consists in transforming cutting-edge innovations in something that can make an impact the current clinical practice. Given the innovative potential of the MOSAIC solutions, we have to analyze in parallel:

1. How the models can be transformed into Decision Support Systems (DSS) for T2DM management and detection (screening tools)

2. Which are the indicators that could testify the success of the MOSAIC solutions

3. Which are the requirements that the end-users would expect from these solutions

For these reasons, the process that will lead to the development and implementation of the system prototype, should involve users and stakeholders in the above analysis. If, from the functional and technical side, the requirements can be gathered by using traditional software engineering techniques like Use Cases definition, other techniques are needed to ensure that the developments will satisfy the needs of the final users and the goals to achieve for the target population. The following 3 Use Cases (UCs) have been defined:

1. UC1: Risk Factors and Indicators to improve current detection of T2DM.

2. UC2: Hospital Care Management.

3. UC3: Clinical Decision Support during follow-up visits.

\section{Methods}

Given the multidisciplinary nature of this project and its challenges, and the need to find a balance on how much effort can be dedicated to the reiteration of the development phases, a holistic strategy is needed. In 2011, the Group from the University of Twente, published in an article called 
"A Holistic Framework to Improve the Uptake and Impact of eHealth Technologies", the CeHRes Roadmap [4.] ¿Error! No se encuentra el origen de la referencia.. This roadmap was built as a result of a systematic review of existing eHealth frameworks, through which the authors gathered the most successful strategies and principles and at the same time, tried to overcome the limitations found. This framework is modular, it is based on a participatory development approach, persuasive design techniques and business modelling and serves as an evidence-based roadmap: it is thus suitable for the MOSAIC purposes and can be used to structure the different activities (research, development, business modelling, validation and evaluation) in a unified strategy. It is a dynamic framework and the authors have also published a wiki for collaborative use (http://ehealthwiki.org).

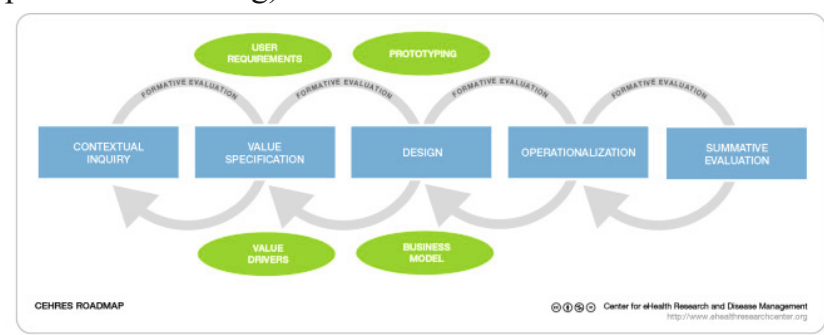

Figure 1 - The CeHRes Roadmap (source: http://www.ehealthresearchcenter.org/wiki/)

The CeHRes Roamap consists of five iterative phases, as shown in the figure. Even though there is a temporal execution of the different phases (from an idea to a product), the iterative nature of this roadmap, the modularity of the different methods and the feedback gathered through the formative evaluation let the user of this method to adapt it to his or her specific needs. The authors provides some guidance on the research methods to be adopted for each case. Below, a brief description of the main methods we have chosen:

\section{- Contextual Inquiry}

Individual and Group Interviews: system users and/or experts discuss together or separately issues of use of the technical system to be developed. Usually, this is done, not only but especially, in the early phases of development, as this reveals which functionalities are more- or less desired, when they are desired and which emotional or organizational problems could be related to them. In the case of MOSAIC, focus groups already took place to discuss the values, attributes and requirements desired. Other focus groups and interviews have been planned, in order to collect some information that had been missing since the start of the project and to gather responses to some concise development questions. This information would be used to produce a prototype which could then be tested by real users.
Analytic Hierarchic Process (AHP), is a multidimensional, multi-level and multifactorial decision-making method based on the idea that it is possible to prioritize elements by: grouping them into meaningful categories and sub-categories; performing pairwise comparison; defining a coherent framework of quantitative and qualitative knowledge; measuring intangible domains. This method has been applied to medical decision-making at hospital level for budget allocation, medical device purchasing and has the potential to be effective for different organizations and individuals interested in eliciting user requirements for example developers wishing to improve device design, hospital managers who must allocate budgets, clinical engineers that are required to select devices.

\section{- Design Phase}

Heuristic analysis and development: usability heuristics, guidelines and standardized design methodology are used in order to identify usability issues in the existing concepts or prototypes of the respective module. As a result, the concept or prototype is refined.

Cognitive walkthrough: this is another usability inspection method but, differently from the heuristic analysis, which takes a holistic view to catch problems, it is taskspecific, focusing on how easy it is for new users to perform the desired tasks.

Usability Tests: this testing by users would be the final step in the usability engineering. Users would complete predefined test tasks. Their behaviour would be recorded and their satisfaction measured with questionnaires. Thus, it would be possible to identify final potential for the improvement of the software prototypes.

Supporting the final pilot tests: in this case, the main objective is to analyse the performance of the system in terms of uptake and impact potential. In the first case, the usage behaviour, the user profile and motivations are assessed, while for the second case, we will look at the impact on healthcare delivery (e.g. efficiency, safety, interaction, etc.) outcomes.

\section{The MOSAIC USER CENTERED DESIGN STRATEGY}

In order to define and develop the User Interface (UI) and Interaction Flows of the MOSAIC system, 4 elements are taken into account:

- The priority assigned to the different values-attributesrequirements, to understand the aspects that need to be tackled most.

- The content, the input and the output of each UI

- The Interaction Flows

- The Components of each UI

\section{- Value Specification}


- The target population of patients (people at risk of T2DM, early T2Dm, T2DM).

All these components will need the involvement of endusers and stakeholders for their definition and validation.

The following steps will be executed for the definition and development of the 3 UCs.

1. The initial starting point was the MOSAIC Grant and the first research activities, where all the modelling techniques, data sources and clinical and technical specifications have been analyzed.

2. Several focus groups where carried out through virtual and physical meetings, as well as informal communications via email, leading to the definition of the main three Use Cases.

3. After that, a low level mock-up (wire framing) is built. This mock-up is evaluated internally and after some iterations a final low level mock-up has been created.

4. Values, attributes and requirements, already extracted through focus groups and workshops, are ranked through the AHP, in order to validate and prioritize the key issues identified so far, resulting in a reinforced user need elicitation.

5. The requirements, are used to create mock-ups and questionnaires that will be validated through focus groups and/or individual interviews, in order to refine the prototype development.

6. Heuristic analysis. The Heuristic Evaluation is carried out, consisting of a session with 4-5 usability experts following the recommendations of the 10 Nielsen Heuristics.

7. The 1st Prototype is analyzed by the consortium partners and then is presented to a small group of experts.

8. The prototype will be analysed through walkthrough and usability tests with end-users.

9. Depending on the above results, the most relevant methods, among the ones described in this manuscript, will be adopted to support the development of the 2nd and final prototype.

\section{Results}

A first iteration has been almost completed (the first 6 points have been executed). We hereby describe the preliminary results coming from the user needs elicitation, heuristic evaluation and concept validation of the first versions of the mockups for each UC.

A. Analytic Hierarchy Process

The following three of needs (or hierarchy) have been created and agreed with the AHP expert and modelling partners.

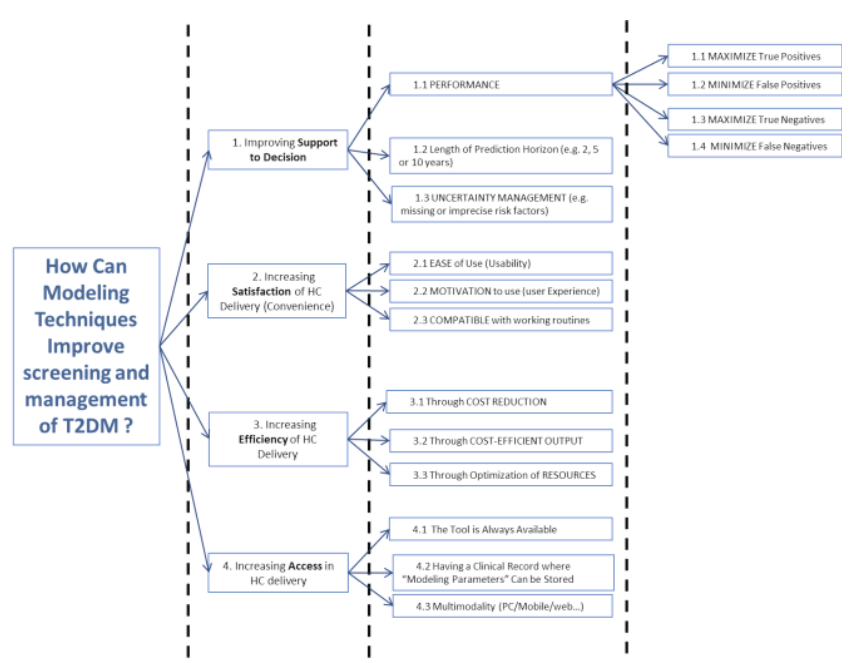

Figure 2 Hierarchy of needs for the development of the MOSAIC Tools

This hierarchy is being currently evaluated by 3 groups of experts ( 1 for each Use Case), among the partners of the consortium. By performing pairwise comparisons, the responders are asked to evaluate which are the most important factors to take into account for developing the tools corresponding to the three main Use Cases. The main questions, for each of them, are the following:

1. In a technological solution for T2DM screening, ...

2. In a technological solution that support you in performing risk stratification on the T2DM population of your clinical department/hospital...

3. In a technological solution that support you in understanding the clinical evolution of your patient...

... and from your point of view and according to your experience, which is the most important element and how much important it is, with respect to the other one (from 1, equally important to 9 much more important)?

To date, two (2) experts have answered to UC1, six (6) to UC2 and three (3) to UC3:

- As regards T2DM screening (UC1), MOSAIC should let improve access in health care delivery, that is, provide a better access to data that are already there but not exploited.

- In the case of the risk stratification for hospital management (UC2), the responders give more importance to increase the support to decision, in particular to minimize false negatives.

- Finally, regarding the support during follow up visits (UC3), users are more interested to increase the efficiency of the health care delivery, with a clear interest in the cost-efficient output component. 
Of course these are preliminary results but it is already interesting to highlight how the same factors are considered differently for the three UCs.

B. User Interfaces Definition and Validation

In this case, given the difficulties in organizing a focus group, it was decided and agreed to validate the interfaces via online questionnaires. These have been created through Google form. For each UC, a description of the application, pictures of the User Interfaces and a video showing the interaction flow have been included.

The same groups and numbers of users answering the AHP questionnaires have been identified for this validation. So far, two (2) experts have answered to UC1, six (6) to UC2 and three (3) to UC3.

In the case of $\mathrm{UC1}$, the main comments are that the tools can be very useful for the working activities of the users, for arranging daily routines. As regards the frequency of use, they suggest to be not on a daily basis or on ("I would close my week in doing 1 hour in the office dedicated with MOSAIC"). Visualizing the path from basic data to recommendation has been required too.

In the case of UC2, the comments about the usefulness of the tools and of the clarity of the information provided are also above the acceptance threshold. Some general charts should be improved in terms of clarity and of relevant information (i.e.: add "cost-opportunity drugs"); the tool should be visualized by all the members of the care team, in order to improve the decision making process.

Finally, for UC3, even though the usefulness and the potential use in clinical practice has been positively rated, users asked to improve the workflow (the evaluation was rated below the acceptance threshold). The decisions they would like to be assisted most are related to the antidiabetic drug prescriptions (patient categorization based on risk of complications) and to the management of both comorbidities and complications.

\section{Heuristic Evaluation of the Interfaces}

The Heuristic Evaluation was carried out by 8 usability experts, to which the same videos and images resources generated previously have been sent. The evaluation is composed of two phases: during the first one, all the responders are asked to carry out a classic Heuristic Evaluation taking into account the Nielsen's Heuristic as reference of the evaluation; in the second one, all the results are merged and this integrated version is sent back to the evaluators who are asked to rate each issue from 0 (not an usability issue) to 4 (very important usability issue).
The results are being used to refine and redefine the interfaces and the interaction flow.

Briefly, the first prototype failed to show clearly the different steps that the users has to fill in in order to achieve a successful outcome, i.e. the approach used in the mock-up based on tabs was not easy to understand for most of the users, instead most of them suggested to use a simple interface showing the number of steps and current step where the user is.

To summarize, the most severe issues regarding the usability were: 1) a severe lack of visibility of system status, especially regarding the different steps that the user has to go through to achieve a correct outcome, 2) according to most of the experts the match between system and the real world should be improved (this involves the employment of a interface closer to the clinical world, both in the written text, icons and so on), 3) a common complaint is that this mock-up did not handle the error prevention, i.e. it did not show how the system would behave in case any error or unexpected behavior happens and finally 4) the system should include more help, documentation and guide the users about how to proceed on each situation according to the experts.

\section{AcKNOWLEDGMENT}

The authors wish to acknowledge the MOSAIC consortium. This project is funded by the European Commission under the 7th Framework Programme, contract n. 600914.

\section{REFERENCES}

[1.] International Diabetes Federation: 5th Edition Diabetes Atlas. Available from http://www.eatlas.idf.org Last accessed April the 10th 2012.

[2.] Diabetes Care. 2004 Jan;27 Suppl 1:S5-S10. Diagnosis and classification of diabetes mellitus. American Diabetes Association.

[3.] American Diabetes Association Consensus Panel. Guidelines for Computer Modeling of Diabetes and its Complications. Diabetes Care September 2004 vol. 27 no. 9 2262-2265

[4.] Van Gemert-Pijnen JEWC, Nijland N, Ossebaard HC, et al. A holistic framework to improve the uptake and impact of eHealth technologies J Med Internet Res. 2011 Oct-Dec; 13(4): e111. Published online Dec 13, 2011. doi: 10.2196/jmir.1672. 